\title{
Puberdade tardia por deficiência de 17alfa-hidroxilase/17-20 liase: Relato de caso
}

\author{
Maria Fernanda M. Fonseca, ${ }^{1, \star}$ Denise L. M. Monteiro ${ }^{2}$
}

\begin{abstract}
Resumo
Introdução: A deficiência de 17alfa-hidroxilase é uma doença autossômica recessiva, causa rara de hiperplasia adrenal congênita, por mutação no gene CYP17A1 que codifica o citocromo P450c17.É caracterizada por infantilismo sexual, hipertensão arterial e hipocortisolismo com aumento dos precursores mineralocorticoides.

Método: Foi realizada ampla pesquisa na literatura nacional e internacional. No Medline (PubMed), foi utilizada a seguinte estratégia de busca: "Puberty, Delayed" AND “Steroid17-alphaHydroxylase" AND "Females", sendo encontrados 16 artigos no total. No SciELO, LILACS e Google Acadêmico encontramos mais 6 artigos. Foram excluídos 3 artigos por abordarem outras síndromes ou por tratarem-se de estudos em animais. A pesquisa bibliográfica finalizou com 19 artigos.

Relato de caso: Adolescente com 17 anos de idade apresentando quadro de amenorreia, ausência de caracteres sexuais secundários e hipertensão arterial. Durante a investigação diagnóstica foram encontrados elevados níveis de gonadotrofinas, progesterona, mineralocorticoides e níveis diminuídos de dehidroepiandrosterona (DHEA) e androstenediona. $\mathrm{O}$ cariótipo foi normal (46, XX). Foi tratada com estrogênio para desenvolvimento mamário. A menarca ocorreu cinco meses após o início do tratamento com dexametasona.
\end{abstract}

Descritores: Hipertensão; Amenorreia; Puberdade tardia.

\section{Abstract \\ Delayed puberty from 17alpha-hydroxylase/17, 20-lyase deficiency: Case report}

Introduction: Deficiency of 17alpha-hydroxylase is an autosomal recessive disease, a rare cause of congenital adrenal hyperplasia, by mutation in the CYP17A1 gene encoding cytochrome P450c17. It is characterized by sexual infantilism, hypertension and hypocortisolism with increased mineralocorticoid precursors.

Methods: An extensive research was carried out in the internacional and national literature. In Medline (PubMed) the following search strategy was adopted: "Puberty, Delayed" AND "Steroid17-alpha-Hydroxylase" AND "Females", resulting in 16 articles. In Scielo, LILACS, and Google Scholar we have found other 6 articles. Three articles were excluded, because the subject was about other different syndromes, or about animal studies. The bibliographical research finished with 19 articles.

Case report: A 17-years-old teenager presenting amenorrhea, absence of secondary characteristics and hypertension. The diagnostic investigation found high levels of gonadotropins,
1. Centro Universitário Serra dos Órgãos. Teresópolis, RJ, Brasil.

2. Professora Adjunta. Faculdade de Ciências Médicas. Universidade do Estado do Rio de Janeiro. Rio de Janeiro, RJ, Brasil.

*Endereço para correspondência:

Av. Professor Manuel de Abreu, 500

CEP: 20550-170. Rio de Janeiro, RJ, Brasil.

E-mail: denimonteiro2@yahoo.com.br

Revista HUPE, Rio de Janeiro, 2016;15(2):187-192

doi: 10.129/rhupe.2016.28245

Recebido em 02/03/2015. Aprovado em 16/03/2016.

progesterone, mineralocorticoid, and decreased levels of dehydroepiandrosterone (DHEA) and androstenedione. The karyotype was normal (46, XX). She was treated with estrogen for breast development. The menarche occurred five months after initiation of treatment with dexamethasone.

Keywords: Hypertension; Amenorrhea; Puberty delayed.

\section{Resumen}

\section{Pubertad ardía por deficiencia de 17 alfa-hidro- xilasa 17-20 liasa: Caso clínico}

Introducción: La deficiencia de 17 alfa-hidroxilasa, enfermedad autosómica recesiva, causa rara de hiperplasia suprarrenal congénita, por mutación en el gen CYP17A1 que codifica el citocromo P450c17. Se caracteriza por infantilismo sexual, hipertensión arterial y hipocortisolismo con un aumento de los precursores de mineralocorticoides.

Método: Se llevó a cabo una amplia investigación en la literatura nacional e internacional. En el Medline (PubMed), se utilizó la siguiente estrategia de búsqueda: "Puberty, Delayed" Y "Steroid 17-alpha-Hydroxylase" Y "Females", encontrándose un total de 16 artículos. En SciELO, LILACS y Google Académico se encontraron seis artículos más. Se excluyeron tres artículos por abordaren otros síndromes o tratarse de estudios con animales. La literatura terminó con 19 artículos.

Caso clínico: Adolescente de 17 años de edad con amenorrea, ausencia de características sexuales secundarias e hipertensión arterial. Durante la investigación diagnóstica se encontraron elevados niveles de gonadotropina, proges- 


\section{Caso clínico}

terona, mineralocorticoides y disminución de los niveles de dehidroepiandrosterona (DHEA) y androstenediona. El cariotipo era normal (46, XX). Se la trató con estrógenos para el desarrollo del pecho. La menarquía se dio cinco meses después del inicio del tratamiento con dexametasona. Palabras clave: Hipertensión; Amenorrea; Pubertad tardía.

\section{Introdução}

A deficiência de 17alfa-hidroxilase P450c é uma rara forma de doença autossômica recessiva que causa diminuição na produção de cortisol e hormônios sexuais e aumento de precursores mineralocorticoides, devido a um defeito no gene CYP17. ${ }^{\text {Apesar de rara, }}$ não é tão incomum em grupos étnicos específicos e em determinadas áreas geográficas, como no Brasil, onde a deficiência de 17alfa-hidroxilase contabiliza 5-7\% de todos os casos de hiperplasia adrenal congênita. ${ }^{2}$ Esta condição foi descrita pela primeira vez por Biglieri e cols. em 1966, em uma mulher adulta (genótipo 46, XX) com hipertensão arterial, hipocalemia e infantilismo sexual. Essa paciente tinha a excreção urinária de corticosterona aumentada, mas com ausência de metabólitos de 17alfa-hidroxilase. ${ }^{3}$

A 17alfa-hidroxilase e a 17-20-liase são responsáveis pela hidroxilação da progesterona e pregnenolona e também pela produção de dehidroepiandrosterona (DHEA) e androstenediona. Se elas estão ausentes, haverá diminuição dos hormônios sexuais e a pregnenolona será convertida em precursores mineralocorticoides. ${ }^{4}$ A ausência de hormônios sexuais cursa com infantilismo sexual, e o aumento de mineralocorticoides pode provocar hipertensão arterial, hipocalemia e alcalose. ${ }^{1,4,5}$

O tratamento é feito por meio da reposição hormonal de estrogênio, estimulando o desenvolvimento sexual e de corticoide para diminuir os níveis de mineralocorticoides. ${ }^{4}$

Pelo fato de essa condição ser extremamente rara, este relato de caso foi realizado com o intuito de lembrar aos ginecologistas a importância do tema como diagnóstico diferencial nos casos de amenorreia primária.

\section{Método}

Para realizar a busca bibliográfica, foi realizada ampla pesquisa na literatura médica procurando informações na literatura nacional e internacional. Para identificação de publicações no Medline (por meio do PubMed), foi utilizada a seguinte estratégia de busca: "Puberty, Delayed" AND “Steroid 17-alpha-Hydroxyla- se" AND "Females", e foram encontrados 16 artigos a partir do ano de 1986. Pesquisamos ainda as bases de dados SciELO, LILACS e Google Acadêmico usando o termo "deficiência de 17alfa- hidroxilase", encontrando um total de 6 artigos. Três artigos foram excluídos por apresentarem outras síndromes ou por se tratarem de estudos em animais. A pesquisa teve um total de 19 fontes bibliográficas.

O projeto de pesquisa foi conduzido dentro dos padrões exigidos pela Declaração de Helsinki e aprovado pelo Comitê de Ética em Pesquisa do Hospital Federal Cardoso Fontes sob o número CAEE 0001.0.374.000-07.

\section{Relato de caso}

FMS, 17 anos, branca, feminina, natural e residente do Rio de Janeiro, procurou o ambulatório de Ginecologia apresentando a queixa de nunca ter menstruado. História de amenorreia primária e ausência de caracteres sexuais secundários. Ao exame, paciente encontrava-se em estágio M1P1 de Tanner, altura de $1,52 \mathrm{~cm}$ e 34,4 kg. Foi solicitada radiografia (RX) de mãos e punhos, que demonstrou idade óssea de 12-13 anos, $\mathrm{FSH}=83,3 \mathrm{mUI} / \mathrm{mL}, \mathrm{LH}=43,2 \mathrm{ng} / \mathrm{mL}$, Prolactina $=28,8$ $\mathrm{ng} / \mathrm{ml}$, progesterona $=10 \mathrm{ng} / \mathrm{mL}, \mathrm{E} 2<20 \mathrm{pg} / \mathrm{mL}$, TSH 2 $\mathrm{mUI} / \mathrm{L}$, ultrassonografia pélvica (USG) evidenciando útero e ovários hipoplásicos.

Os altos índices de FSH e LH suscitaram a hipótese de disgenesia gonadal. Prosseguiu-se a investigação, e verificou-se cariótipo normal (46, XX). Encontraram-se níveis baixos de androgênios: DHEA-S=1,39 mcg/dL ( N= $34-430 \mathrm{mcg} / \mathrm{dL}$ ), testosterona $=0,1 \mathrm{pg} / \mathrm{mL}(\mathrm{N}=\mathrm{F}$. Folicular: 0,45-3,17 pg/mL; F. Lútea: 0,46-2,48), 17OH progesterona $0,1 \mathrm{ng} / \mathrm{mL}$ ( $\mathrm{N}=$ F. Folicular: 0,35-1 ng/mL; F. Lútea: $1,3-3,6 \mathrm{ng} / \mathrm{dL})$, androstenediona $=0,4 \mathrm{ng} / \mathrm{ml}(\mathrm{N}=0,8-4,4$ $\mathrm{ng} / \mathrm{mL}$ ). As tomografias computadorizadas de sela turca e suprarrenal foram normais. A paciente foi submetida à videolaparoscopia evidenciando-se útero e ovários hipotróficos. Este fato motivou a realização de biopsia ovariana, cujo laudo histopatológico demonstrou parênquima ovariano com camada cortical contendo folículos primordiais e alguns folículos secundários. No pré-operatório foi identificada hipertensão arterial (160x100mmHg).

Realizou-se a dosagem de cortisol= $0,6 \mu \mathrm{g} / \mathrm{dL}(\mathrm{N}=$ 5-25), que por mostrar nível reduzido indicou a realização do teste do ACTH, cujos resultados encontram-se na tabela 1 .

Ocortisol pós-ACTH deve ser $>20 \mu \mathrm{g} / \mathrm{dL}$ e neste caso não houve aumento. O resultado do ACTH foi de 487,1 $\mathrm{pg} / \mathrm{mL}(\mathrm{N}=10-60)$, corticosterona $=13,321 \mathrm{ng} / \mathrm{dL}(\mathrm{N}=100$ - 
$700 \mathrm{ng} / \mathrm{dL}$ - teste realizado nos EUA) e 11 desoxicortisol= 0,6 ng/mL (após teste do ACTH, aumento de 3-5x).

Durante a investigação diagnóstica, iniciou-se tratamento com estrogênios conjugados (EC) $0,625 \mathrm{mg} / \mathrm{dia}$ com aparecimento do broto mamário após três meses de medicação. Oito meses depois, aumentou-se a dose de para 1,25 mg/dia. Após a vídeolaparoscopia e o teste do ACTH, foi iniciada dexametasona $0,75 \mathrm{mg} / \mathrm{dia}$. A menarca ocorreu cinco meses depois, aos 19 anos de idade.

Dois anos após a primeira menstruação, a paciente encontrava-se ao exame físico com altura de 1,67 cm, peso de $42 \mathrm{~kg}$, com ciclos menstruais irregulares e Tanner M2 P3.

\section{Discussão}

Nossa paciente apresenta um quadro de amenorreia associada à ausência de caracteres sexuais secundários, aos 17 anos, o que caracteriza um quadro de puberdade tardia.

Amenorreia é a ausência de menstruação durante o menacme. A menstruação é o que define integridade do eixo hipotálamo-hipófise-gonadal e a funcionalidade de útero e ovários. Pode ser dividida, de acordo com o critério cronológico, em amenorreia primária (prevalência de 0,3-0,5\%) e secundária (prevalência de 5\%). ${ }^{6}$

Amenorreia primaria é a ausência de menstruação em uma menina com ausência de caracteres sexuais aos 12 anos, aos 16 com desenvolvimento puberal normal ou após seis anos do aparecimento do broto mamário. Já amenorreia secundária é a ausência de menstruação por um período igual ou superior a três ciclos menstruais consecutivos em mulheres com ciclos anteriores regulares ou por seis meses em mulheres que já apresentaram uma menstruação espontânea anterior e ciclos irregulares. ${ }^{6}$

Na grande parte dos casos, a amenorreia primária é causada por alterações gonadais (insuficiência ovariana primária), anatômicas, ou centrais (hipófise e hipotálamo). ${ }^{6}$

O primeiro passo na avaliação de um quadro de amenorreia é a anamnese e o exame físico detalhados. ${ }^{6}$ Diante do quadro clínico apresentado pela nossa paciente, a conduta é a dosagem de gonadotrofinas. O resultado do exame evidenciou níveis aumentados de FSH e LH, o que fortificou a hipótese de disgenesia gonadal.

A disgenesia gonadal ocorre por anomalias dos cromossomas sexuais ou por mutações em genes envolvidos na diferenciação sexual. Nesses casos, as gônadas passam a não responder aos estímulos de FSH e LH, havendo aumento na concentração de gonadotrofinas e diminuição de estrogênio e testosterona, ${ }^{7}$ quadro laboratorial compatível com o apresentado pela paciente.

Com o objetivo de identificar o subtipo de disgenesia, solicitamos o cariótipo, cuja normalidade (46, XX), levou-nos à conclusão de que se tratava de disgenesia gonadal pura.

Vale ressaltar que o diagnóstico dessa condição é realizado pela dosagem de FSH/LH, cariótipo e USG de pelve (ovários em fita).7 O laudo histopatológico demonstrou que os ovários apresentavam folículos em vários estágios do desenvolvimento, o que excluiu totalmente a possibilidade de disgenesia gonadal.

Diante da situação acima descrita, além da constatação da hipertensão arterial, foram realizados novos exames que confirmaram a diminuição de hormônios sexuais e de cortisol e aumento de ACTH e gonadotrofinas, o que, após buscas na literatura, nos direcionou para o diagnóstico de deficiência de 17alfa-hidroxilase.

A deficiência de 17alfa-hidroxilase P450c, como citado anteriormente, é uma forma de hiperplasia adrenal congênita rara (menos de $1 \%$ dos casos) que causa diminuição na produção de cortisol e hormônios sexuais e o aumento na síntese de precursores mineralocorticoides. ${ }^{1,8}$ Kim e cols. relataram um caso de um paciente coreano que, apesar de longo tempo apresentando hipertensão e amenorreia primária, só foi diagnosticada aos 42 anos, mostrando que, devido à raridade, o diagnóstico geralmente é tardio, ocorrendo na maioria dos casos, na época da puberdade fisiológica. ${ }^{9}$

É causada por uma mutação no gene CYP17A1 citocromo P450c17, enzima expressa no córtex da adrenal e nas gônadas, com atividade de 17 alfa-hidroxilase e 17,20-liase. ${ }^{1,10} \mathrm{~A}$ mutação genética no CYP17A1 afeta tanto a esteroidogênese ovariana quanto adrenal. ${ }^{11} \mathrm{~A}$ 17alfa-hidroxilase e a 17-20-liase são responsáveis pela hidroxilação da progesterona e pregnenolona e pela produção de DHEA e androstenediona.,11,12 Se nem a atividade da 17alfa-hidroxilase nem da 17,20-liase estão presentes, a pregnenolona é convertida em mineralocorticoide. Se apenas a 17alfa-hidroxilase está presente, a pregnenolona é convertida em cortisol, e se ambas as atividades enzimáticas estão presentes, a pregnenolona é convertida em DHEA.,13 Sendo assim, a deficiência de P450c17 leva à diminuição da síntese de cortisol e esteroides sexuais, com aumento nos níveis circulantes de ACTH e, consequentemente, de deoxicorticosterona (DOC), corticosterona e 18OH-corticosterona (Figura 1), ${ }^{1,4}$

A falha na produção de hormônios sexuais nos pacientes com deficiência de 17alfa-hidroxilase/17-20-liase 


\section{Caso clínico}

resulta em infantilismo sexual, amenorreia primária e ausência de pelos pubianos e axilares nas mulheres (46, XX), ${ }^{4,5,11}$ quadro clínico apresentado pela paciente em questão. Em adição, a diminuição nos níveis de cortisol, leva a um aumento na secreção de ACTH que estimula a superprodução de corticosterona e deoxicorticosterona. ${ }^{5,14}$ Os altos níveis de DOC causam diminuição da renina e aumento da produção de aldosterona, sendo os responsáveis pelo quadro hipertensivo e pela hipocalemia ${ }^{8,15,16}$ No caso relatado, a paciente apresentava esse quadro clínico característico. Uma minoria, menos de 10\%, apresenta níveis normais de pressão arterial e de potássio na hora do diagnóstico. ${ }^{17}$

Em homens, o defeito enzimático manifesta-se por quadro de pseudo-hermafroditismo masculino (46 XY), com genitália feminina, vagina em fundo cego e ausência de estruturas müllerianas. ${ }^{4,8,15,16} \mathrm{New}^{16} \mathrm{fez}$ o primeiro relato de deficiência de 17alfa-hidroxilase em homens. No seu relato, ela descreve um caso de pseudo-hermafroditismo, genitália externa ambígua, ausência de caracteres sexuais masculinos secundários e ginecomastia na puberdade. Assim como a nossa paciente, o caso estudado por ela apresentava baixos níveis de cortisol e elevados níveis de deoxicorticosterona e corticosterona, mas o cariótipo encontrado foi 46, XY. O paciente não apresentava hipertensão e hipocalemia graves. Diante do encontrado, New verificou que não há diferença no padrão laboratorial entre os gêneros. Biglieri e cols. também descreveram um caso semelhante, mas com padrão clínico mais grave. Ambos os estudos verificaram que a hipertensão está ligada aos altos níveis de aldosterona, fato comprovado pela sua normalização após administração de dexametasona. Kim e cols. relataram uma paciente que, geneticamente era homem (46, XY), e que possuía genitália completa feminina. Apresentava-se aos 42 anos de idade e desde os 30 fazia tratamento para controle de pressão arterial sem sucesso., ${ }^{9,14}$

Alguns pacientes apresentam deficiência parcial de

Tabela 1. Teste da cortrosina ( $250 \mu \mathrm{g}$ ACTH sintético).

\begin{tabular}{|l|c|c|}
\hline & BASAL & APÓs $60 \mathrm{MINUTOS}$ \\
\hline Cortisol & $0,7 \mu \mathrm{g} / \mathrm{dL}$ & $0,6 \mu \mathrm{g} / \mathrm{dL}$ \\
\hline $170 H$ progesterona & $98,6 \mathrm{ng} / \mathrm{dL}$ & $95,9 \mathrm{ng} / \mathrm{dL}$ \\
\hline Androstenediona & $<40 \mathrm{pg} / \mathrm{mL}$ & $<40 \mathrm{pg} / \mathrm{mL}$ \\
\hline Progesterona & $8200 \mathrm{pg} / \mathrm{mL}$ & $5732 \mathrm{pg} / \mathrm{mL}$ \\
\hline
\end{tabular}

17 alfa-hidroxilase com algum grau de síntese de hormônios sexuais, levando a quadros de genitália ambígua (em homens genéticos), como encontrado por New, e irregularidade menstrual (em mulheres genéticas). ${ }^{4} \mathrm{~A}$ gravidade da ausência de virilização está relacionada com a gravidade do defeito enzimático. ${ }^{18}$

Outras manifestações secundárias à diminuição na produção de hormônios sexuais como alta estatura, osteoporose e retardo da idade óssea podem ocorrer. ${ }^{4}$ Nossa paciente apresentava idade óssea de 11-12 anos, quando a idade cronológica era de 17 anos. Müssig e cols. relataram o caso de uma paciente com quadro de amenorreia, ausência de caracteres secundários e osteoporose. A paciente não apresentava atividade nem de 17 alfa-hidroxilase nem de 17-20-liase e, para o tratamento da osteoporose, foi usado colecalciferol e cálcio. ${ }^{10}$

A insuficiência de glicocorticoides clinicamente é rara, apesar da diminuição de cortisol, já que a excessiva produção de corticosterona promove adequada resposta a situações de estresse., ${ }^{1,411} \mathrm{O}$ diagnóstico diferencial de hipocalemia, hipertensão e atraso puberal inclui tanto doenças renais quanto doenças endócrinas. As doenças a serem consideradas são síndrome de Liddle, síndrome do excesso aparente de mineralocorticoide, e outras causas incomuns de hiperplasia adrenal congênita. Nos casos de doença primária renal, a puberdade tardia pode ser resultado de uma doença crônica. ${ }^{1}$

No processo de investigação da paciente, o padrão laboratorial encontrado, como citado no relato, foi o descrito na literatura: níveis de gonadotrofinas, de progesterona, deoxicorticosterona, corticosterona e pregnenolona bastante aumentados, ao passo que os níveis de androstenediona, DHEA, testosterona e estradiol estavam diminuídos. ${ }^{4,17}$ Os níveis de ACTH encontrados após teste com cortrosina estavam aumentados, porém mais baixos do que em outras doenças que diminuem o cortisol. Esses achados corroboram o diagnóstico de deficiência de 17alfa-hidroxilase.

O estudo anatomopatológico das glândulas adrenais mostra hiperplasia cortical difusa, hiperplasia nodular ou hiperplasia adenomatosa. A histologia ovariana de mulheres acometidas pelo infantilismo sexual mostra ovários contendo folículos em diferentes estágios de desenvolvimento. ${ }^{4}$ Já nos casos de disgenesia gonadal, os ovários são hipoplásicos, em fita e com atresia folicular acelerada.

Diferentemente do perfil dos esteroides no soro que são bem estudados, a avaliação dos seus metabolitos urinários é bem limitada. ${ }^{5}$ Foi realizado um estudo que avaliou os níveis de esteroides no soro e os metabolitos 
esteroides urinários de 20 pacientes que apresentavam deficiência completa de 17-hidroxilase devido a mutações especificas no gene CYP17A1 (W406R e R362C). ${ }^{5}$ Esse estudo confirmou estudos anteriores que encontraram níveis aumentados de corticosterona e deoxicorticosterona e seus metabolitos - $18 \mathrm{OHB}$ e 18OHDOC. Também confirmou a dissociação de $18 \mathrm{OHD}$ na presença de aldosterona reduzida, explicada pela produção de $18 \mathrm{OH}$ na zona fasciculada do seu precursor corticosterona, estimulada pelo aumento na produção de ACTH. ${ }^{5}$

A progesterona elevada na presença de hipogonadismo hipergonadotrófico é uma forte evidência de diagnóstico de deficiência de 17 alfa-hidroxilase. ${ }^{5}$ Segundo Croxson e cols., a elevação inesperada desse hormônio é uma pista para a presença de aumento dos precursores de esteroides e o verdadeiro diagnóstico. ${ }^{19}$

Mulheres com infantilismo sexual, como a paciente do caso, precisam de estrogênio para induzir o desenvolvimento mamário e os caracteres sexuais secundários.1,14 Ele é tipicamente iniciado no tempo esperado para o início da puberdade. Pode ser usado estrogênio conjugado, no início, em baixas doses. Após a ocorrência de menstruação ou 24 meses da reposição de estrogênio, a progesterona oral é introduzida para estabelecer ciclos menstruais regulares. ${ }^{1}$ Após o uso dessa terapia hormonal, a paciente passou a apresentar desenvolvimento sexual secundário e teve sua primeira menstruação.

A administração de glicocorticoides é usada para di- minuir o ACTH e, com isso, os níveis de corticosterona e deoxicorticosterona. Assim, corrige-se também a hipertensão arterial, que pode ser refratária, principalmente se existente há longo tempo. ${ }^{1,11,14,15} \mathrm{~A}$ definição do sexo é baseada nas características anatômicas e fisiológicas da genitália externa mais que no sexo genético. ${ }^{4}$

\section{Conclusão}

A deficiência de 17alfa-hidroxilase é pouco prevalente e a literatura a respeito é escassa. Não faz parte da investigação inicial do ginecologista diante de um quadro de amenorreia primária, tornando-se, então, um diagnóstico tardio. Assim, esse relato de caso foi realizado com o intuito de chamar a atenção para a importância de uma condição rara, que deve ser lembrada como diagnóstico diferencial, e mostrar que a detecção precoce possibilita o início da terapia hormonal na época da puberdade, minimizando os efeitos orgânicos decorrente da falta de hormônios sexuais e do excesso de precursores mineralocorticoides.

\section{Referências}

1. Olson CA, Crudo DF. Pubertal delay, hypokalemia, and hypertension caused by a rare form of congenital adrenal hyperplasia. J Pediatr Adolesc Gynecol. 2011;24(2):e29-31. doi:10.1016/j.jpag.2010.08.018.

2. Miller WL. Steroid 17alpha-hydroxylase deficiency not rare everywhere. J Clin Endocrinol Metab. 2004;89(1):40-2. http:// dx.doi.org/10.1210/jc.2003-031770.

3. Brooke AM, Taylor NF, Shepherd JH, et al. A novel point mutation in P450c17 (CYP17) causing combined 17alpha-hydrox-
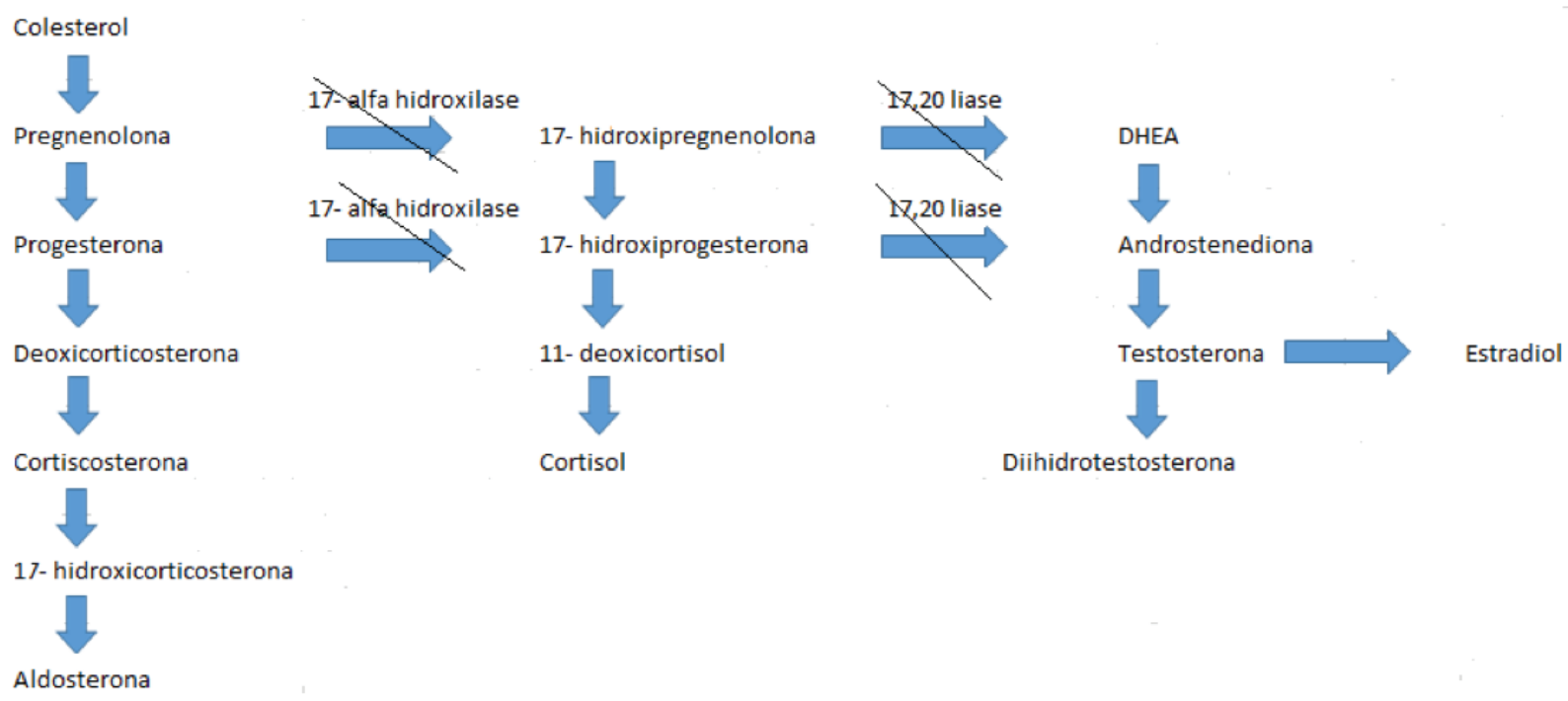

Figura 1. Esteroidogênese ovariana.

Fonte: modificada de Olson et.al, 2011. 


\section{Caso clínico}

ylase/17,20-lyase deficiency. J Clin Endocrinol Metab. 2006 91(6):2428-31.

4. Silva EA, Siqueira ACL, Pacheco S, et al. Hiperplasia adrenal congênita-deficiência de 17-alpha-hidroxilase. Arq Bras Endocrinol Metab. 2000;44(2):175-7.

5. Neres MS, Auchus RJ, Shackleton CHL, et al. Distinctive profile of the 17-hydroxylase and 17,20-lyase activities revealed by urinary steroid metabolomes of patients with CYP17 deficiency. Arq Bras Endocrinol Metab. 2010;54(9):826-32. http://dx.doi. org/10.1590/S0004-27302010000900009.

6. Klein DA, Poth MA. Amenorrhea: an approach to diagnosis and management. American Family Physician. 2013;87(11):781-8. Disponível em www.aafp.org/afp. Acessado em 15Jul2015.

7. Castro LC, Rodrigues RG, Charone DM, et al. Disgenesia gonadal pura X: Relato de caso. Rev para med. 2013;27(1). Disponível em http://files.bvs.br/ upload/S/0101-5907/2013/ v27n1/a3534.pdf. Acessado em 17Jul2015.

8. Auchus RJ. The genetics, pathophysiology, and management of human deficiencies of P450c17. Endocrinol Metab Clin North Am. 2001;30(1):101-19.

9. Kim YM, Kang M, Choi JH, et al. A review of the literature on common CYP17A1 mutations in adults with17-hydroxylase/17,20-lyase deficiency, a case series of such mutations among Koreans and functional characteristics of a novel mutation. Metabolism. 2014;63(1):42-9. doi: 10.1016/j.metabol. 2013.08.015.

10. Müssig K, Kaltenbach S, Machicao F, et al. 17alpha-hydroxylase/17,20-lyase deficiency caused by a novel homozygous mutation (Y27Stop) in the cytochrome CYP17 gene. J Clin Endocrinol Metab. 2005;90(7):4362-5.
11. Costernaro F, Rodrigues TC, Kater CE, et al. Combined 17-alpha-hydroxylase/17,20-lyase deficiency due to p.R96W mutation in the CYP17 gene in a Brazilian patient. Arq Bras Endocrinol Metabol. 2010;54(8):711-6. http://dx.doi.org/10.1590/ S0004-27302010000800014.

12. Rosa S, Duff C, Meyer M, et al. P450c17 deficiency: clinical and molecular characterization of six patients. J Clin Endocrinol Metab. 2007;92(3):1000-7.

13. Belgini DRB, Mello MP, Baptista MTM, et al. Seis novos casos confirmam o perfil clínico molecular de deficiência combinada de 17 alfa-hidroxilase/17,20-liase no Brasil. Arq Bras Endocrinol Metabol. 2010;54(8):711-6. http://dx.doi.org/10.1590/ S0004-27302010000800008.

14. Kim SM, Rhee JH. A case of 17 alpha-hydroxylase deficiency. Clin Exp Reprod Med. 2015;42(2):72-6. doi: 10.5653/ cerm.2015.42.2.72.

15. Biglieri EG, Herron MA, Brust N. 17-Hydroxylation Deficiency in Man. J Clin Invest. 1996;45(12):1946-5.

16. New MI. Male pseudohermaphroditism due to 17-alpha-hydroxylase deficiency. J Clin Invest. 1970;49(10):1930-41. doi: 10.1172/JCl106412.

17. Karter CE, Biglieri EG. Disorders of steroid 17alpha-hydroxilase deficiency. Endocrinol Metab Clin North Am. 1994;23:341-7.

18. Kok RC, Timmerman MA, Wolffenbuttel KP, et al. Isolated 17,20 -lyase deficiency due to the cytochrome b5 mutation W27X. J Clin Endocrinol Metab. 2010;95(3):994-9.

19. Croxson M, Ogilvie CM, Milsom S, et al. Delayed puberty from partial 17-alpha hydroxylase enzyme deficiency. N Z Med J. 2012;125(1355):71-4. 